\title{
A RESULT CONCERNING DERIVATIONS IN BANACH ALGEBRAS
}

\author{
J. VUKMAN
}

(Communicated by Palle E. T. Jorgensen)

\begin{abstract}
The main result: Let $A$ be a Banach algebra over the complex field $C$. Suppose there exists a continuous derivation $D: A \rightarrow A$, such that $\alpha D^{3}+D^{2}$ is a derivation for some $\alpha \in C$. In this case $D$ maps $A$ into its radical.
\end{abstract}

This paper is a continuation of our earlier work [7,8]. Let $R$ be a ring with center $Z(R)$. We write $[x, y]$ for $x y-y x$ and use the identities $[x y, z]=$ $[x, z] y+x[y, z],[x, y z]=[x, y] z+y[x, z]$. An additive mapping $D: R \rightarrow R$ is called a derivation if $D(x y)=D(x) y+x D(y), x, y \in R$. A derivation $D$ is inner if there exists $a \in R$, such that $D(x)=[a, x]$ holds for all $x \in R$. Recall that a ring $R$ is prime if $a R b=(0)$ implies that either $a=0$ or $b=0$, and is semiprime if $a R a=(0)$ implies $a=0$.

The main purpose of this paper is to prove the following result.

Theorem 1. Let $A$ be a Banach algebra over the complex field $C$. Suppose there exists a continuous derivation $D: A \rightarrow A$, such that $\alpha D^{3}+D^{2}$ is a derivation for some $\alpha \in C$. In this case $D$ maps $A$ into its radical.

In the proof of Theorem 1 we need the result below, which might be of some independent interest. The proof of this purely algebraic result is rather long, but it is elementary in the sense that one can follow the proof without any specific knowledge concerning prime rings.

Theorem 2. Let $R$ be a prime ring of characteristic different from two. Suppose there exists derivations $D_{1}, D_{2}$, and $D_{3}$ on $R$, such that $D_{1}\left(D_{1}^{2}(x)+D_{2}(x)\right)=$ $D_{3}(x)$ holds for $x \in R$. In this case we have either $D_{1}=0$ or $D_{2}=0$.

Proof. Let us introduce a mapping $F: R \rightarrow R$ by the relation $F(x)=D_{1}^{2}(x)+$ $D_{2}(x)$. A routine calculation shows that

$$
F(x y)=F(x) y+x F(y)+2 D_{1}(x) D_{1}(y)
$$

Received by the editors November 12, 1990.

1980 Mathematics Subject Classification (1985 Revision). Primary 46H05, 46J05, 47B47; Secondary 16A12, 16A72.

Key words and phrases. Banach algebra, commutative Banach algebra, $C^{*}$-algebra, prime ring, semiprime ring, derivation, inner derivation.

This research has been supported by the Research Council of Slovenia, Yugoslavia.

(C) 1992 American Mathematical Society $0002-9939 / 92 \$ 1.00+\$ .25$ per page 
holds for all pairs $x, y \in R$. Now the assumption of the theorem can be written in the form

$$
D_{1}(F(x))=D_{3}(x), \quad x \in R .
$$

Let $x$ be $x y$ in (2). Then according to (1), we have

$$
\begin{aligned}
0= & D_{1}(F(x y))-D_{3}(x y) \\
= & D_{1}\left(F(x) y+x F(y)+2 D_{1}(x) D_{1}(y)\right)-D_{3}(x) y-x D_{3}(y) \\
= & D_{1}(F(x)) y+F(x) D_{1}(y)+D_{1}(x) F(y)+x D_{1}(F(y)) \\
& +2 D_{1}^{2}(x) D_{1}(y)+2 D_{1}(x) D_{1}^{2}(y)-D_{3}(x) y-x D_{3}(y) .
\end{aligned}
$$

According to (2), the above calculation reduces to

$$
F(x) D_{1}(y)+D_{1}(x) F(y)+2 D_{1}^{2}(x) D_{1}(y)+2 D_{1}(x) D_{1}^{2}(y)=0,
$$

which can be written in the form

$$
H(x) D_{1}(y)+D_{1}(x) H(y)=0, \quad x, y \in R,
$$

where $H(x)$ stands for $3 D_{1}^{2}(x)+D_{2}(x)$. It is easy to see that

$$
H(x y)=H(x) y+x H(y)+6 D_{1}(x) D_{1}(y)
$$

holds for all pairs $x, y \in R$. Let $y$ be $y z$ in (3). Then according to (4), we have

$$
\begin{aligned}
0= & H(x) D_{1}(y z)+D_{1}(x) H(y z) \\
= & H(x) D_{1}(y) z+H(x) y D_{1}(z)+D_{1}(x) H(y) z \\
& +D_{1}(x) y H(z)+6 D_{1}(x) D_{1}(y) D_{1}(z) .
\end{aligned}
$$

Since (3) holds, the above calculation gives

$$
H(x) y D_{1}(z)+D_{1}(x) y H(z)+6 D_{1}(x) D_{1}(y) D_{1}(z)=0, \quad x, y, z \in R .
$$

For $y=D_{1}(x)$ and $z=y$ the relation (5) gives

(6) $H(x) D_{1}(x) D_{1}(y)+D_{1}(x)^{2} H(y)+6 D_{1}(x) D_{1}^{2}(x) D_{1}(y)=0, \quad x, y \in R$.

Left multiplication of $(3)$ by $D_{1}(x)$ gives

$$
D_{1}(x) H(x) D_{1}(y)+D_{1}(x)^{2} H(y)=0, \quad x, y \in R .
$$

Combining (6) with (7) one obtains

$$
\left(H(x) D_{1}(x)-D_{1}(x) H(x)\right) D_{1}(y)+6 D_{1}(x) D_{1}^{2}(x) D_{1}(y)=0 .
$$

In the above relation, one can replace $H(x) D_{1}(x)-D_{1}(x) H(x)$ by $-2 D_{1}(x) H(x)$ because of (3). Thus, since $R$ is not of characteristic 2 , we have

$$
D_{1}(x)\left(H(x)-3 D_{1}^{2}(x)\right) D_{1}(y)=0
$$

and finally

$$
D_{1}(x) D_{2}(x) D_{1}(y)=0, \quad x, y \in R,
$$

because $H(x)=3 D_{1}^{2}(x)+D_{2}(x)$. Putting $z y$ instead of $y$ in (8) we arrive at

$$
D_{1}(x) D_{2}(x) z D_{1}(y)=0, \quad x, y, z \in R \text {. }
$$


From the above relation, one obtains that either $D_{1}=0$ or $D_{1}(x) D_{2}(x)=0$ for all $x \in R$. In any case

$$
D_{1}(x) D_{2}(x)=0, \quad x \in R .
$$

The linearization of (9) leads to

$$
D_{1}(x) D_{2}(y)+D_{1}(y) D_{2}(x)=0, \quad x, y \in R .
$$

Putting $y z$ instead of $y$ in (10) we obtain using (10) again

$$
\begin{aligned}
0 & =D_{1}(x) D_{2}(y z)+D_{1}(y z) D_{2}(x) \\
& =D_{1}(x) D_{2}(y) z+D_{1}(x) y D_{2}(z)+D_{1}(y) z D_{2}(x)+y D_{1}(z) D_{2}(x) \\
& =-D_{1}(y) D_{2}(x) z+D_{1}(x) y D_{2}(z)+D_{1}(y) z D_{2}(x)-y D_{1}(x) D_{2}(z) .
\end{aligned}
$$

Thus we have

$$
D_{1}(y)\left[z, D_{2}(x)\right]+\left[D_{1}(x), y\right] D_{2}(z)=0,
$$

and, in particular, for $z=D_{2}(x)$

$$
\left[D_{1}(x), y\right] D_{2}^{2}(x)=0, \quad x, y \in R .
$$

Substituting $y z$ for $y$ in (12), we obtain

$$
\left[D_{1}(x), y\right] z D_{2}^{2}(x)=0, \quad x, y, z \in R .
$$

Suppose that $D_{2}^{2}(a) \neq 0$ for some $a \in R$. In this case it follows from (13) that $\left[D_{1}(a), y\right]=0, y \in R$, by primeness of $R$. In other words $D_{1}(a) \in Z(R)$. Now putting $x=a$ in (9) and multiplying (9) from the left by $x$, we obtain

$$
D_{1}(a) x D_{2}(a)=0, \quad x \in R,
$$

whence it follows that $D_{1}(a)=0$, otherwise $D_{2}(a)$ would be zero, which contradicts the assumption $D_{2}^{2}(a) \neq 0$. Now for $y=a$, the relation (10) reduces to $D_{1}(x) D_{2}(a)=0, x \in R$, whence putting $x=a$ in (11) we obtain

$$
D_{1}(x) y D_{2}(a)=0, \quad x, y \in R,
$$

which implies $D_{1}=0$, since $D_{2}(a) \neq 0$. Therefore we have proved that $D_{1}=0$ in case $D_{2}^{2}(a) \neq 0$ for some $a \in R$. In other words $D_{2}^{2}(x)=0$ for all $x \in R$ if $D_{1} \neq 0$. But in this case we have $D_{2}=0$ by Theorem 1 in [3]. The proof of the theorem is complete.

Theorem 2 is in the spirit of a classical result of Posner [3, Theorem 1], which states that if $R$ is a prime ring of a characteristic different from two and $D$, $G$ are nonzero derivations of $R$, then $D G$ cannot be a derivation.

Proof of Theorem 1. Sinclair [4] has proved that any continuous linear derivation of a Banach algebra leaves the primitive ideals invariant. Since the radical of $A$ is the intersection of all primitive ideals, any continuous linear derivation leaves the radical invariant as well, which means that there is no loss of generality in assuming that $A$ is semisimple. Since $D$ leaves all primitive ideals invariant, one can introduce for any primitive ideal $P \subset A$ a derivation $D_{P}: A / P \rightarrow A / P$, where $A / P$ is the factor algebra, by $D_{P}(\hat{x})=D(x), \hat{x}=x+P$. Singer and Wermer [5] have proved that any continuous linear derivation on a commutative Banach algebra maps the algebra into its radical. By the result of Johnson and Sinclair [2], any linear derivation on a semisimple Banach algebra is continuous. 
Hence there is no nonzero linear derivation on a commutative Banach algebra. Therefore in case $A / P$ is a commutative Banach algebra, one can conclude that $D_{P}=0$, because $A / P$ is semisimple. It remains to prove that $D_{P}=0$; also in the case, $A / P$ is noncommutative. The assumption of the theorem that $\alpha D^{3}+D^{2}$ is a derivation gives that $\alpha D_{P}^{3}+D_{P}^{2}$ is a derivation. Let us first assume that $\alpha=0$. In this case we have $D_{P}^{2}$ is a derivation, and since $A / P$ is prime, it follows from [2, Theorem 1] that $D_{P}=0$. In case $\alpha \neq 0$ all the assumptions of Theorem 2 are fulfilled (note that $D_{P}$ stands for $D_{1}$ and $D_{P} / \alpha$ for $D_{2}$ ). Thus we have $D_{P}=0$ or $D_{P} / \alpha=0$. In any case $D_{P}=0$. In other words, $D(x)$ is in the intersection of all primitive ideals of $A$ for any $x \in A$. Since the intersection of all primitive ideals is the radical, the proof of the theorem is complete.

In the proof of Theorem 1 we used the Singer-Wermer theorem, which states that any continuous linear derivation on a commutative Banach algebra maps the algebra into its radical. Thomas [6] has generalized the Singer-Wermer theorem by proving that the continuity assumption is superfluous. This leads to the question of whether Theorem 1 can be proved without any continuity assumptions. We do not know whether the answer to this question is affirmative. However, in a special case when a Banach algebra is semisimple, one can prove the following result.

Theorem 3. Let $A$ be a semisimple Banach algebra over the complex field $C$. Suppose there exists a linear derivation $D: A \rightarrow A$, such that $\alpha D^{3}+D^{2}$ is a derivation for some $\alpha \in C$. In this case $D=0$

Proof. The proof goes through in the same way as the proof of Theorem 1 with the only exception that at the beginning of the proof one has to use the fact that any linear derivation on a semisimple Banach algebra is continuous (see [4]).

It is interesting that in Theorem 3 the $\alpha=0$ case is included; because it is known (see the proof of Lemma 1.1.9 in [1]) that if $D$ and $D^{2}$ are derivations of a semiprime ring (in particular a $C^{*}$-algebra), then $D=0$. Let us point out that one can prove nothing in case $D$ and $D^{3}$ are derivations. Namely if one takes $a$, which is not in the center such that $a^{2}=0, D(x)=[a, x]$, then an easy calculation shows that $D^{3}(x)=0$ for all $x$, but $D \neq 0$.

\section{ACKNOWLEDGMENT}

The author wishes to express his thanks to Dr. Matej Brešar for helpful conversations while this paper was being prepared. He also thanks the referee for helpful suggestions.

\section{REFERENCES}

1. I. N. Herstein, Rings with involution, Chicago Lectures in Math., Univ. of Chicago Press, Chicago and London, 1976.

2. B. E. Johnson and A. M. Sinclair, Continuity of derivations and a problem of Kaplansky, Amer. J. Math. 90 (1968), 1067-1073.

3. E. Posner, Derivations in prime rings, Proc. Amer. Math. Soc. 8 (1957), 1093-1100.

4. A. M. Sinclair, Continuous derivations on Banach algebras, Proc. Amer. Math. Soc. 20 (1969), 166-170. 
5. I. M. Singer and J. Wermer, Derivations on commutative normed algebras, Math. Ann. 129 (1955), 260-264.

6. M. P. Thomas, The image of a derivation is contained in the radical, Ann. of Math. (2) 28 (1988), 435-460.

7. J. Vukman, Commuting and centralizing mappings in prime rings, Proc. Amer. Math. Soc. 109 (1990), 47-52.

8. _ A result concerning derivations in noncommutative Banach algebras, Glas. Mat. Ser. III (to appear).

Department of Mathematics, UNiversity of Maribor, PF, Koroška 160, 62000 Maribor, YugosLAVIA 